\title{
Severe leukoencephalopathy with fulminant cerebral edema reflecting immune reconstitution inflammatory syndrome during HIV infection: a case report
}

Christian Oelschlaeger*1, Rainer Dziewas¹, Doris Reichelt², Jens Minnerup¹, Thomas Niederstadt³ , Erich B Ringelstein and Ingo W Husstedt ${ }^{1}$

\begin{abstract}
Introduction: Immune reconstitution inflammatory syndrome is a well-known complication in HIV-infected patients after initiation of highly active antiretroviral therapy resulting in rapid $C D 4^{+}$cell count recovery and suppression of viral load. Generally, immune reconstitution inflammatory syndrome is based on opportunistic infections, but rare cases of immune reconstitution inflammatory syndrome inducing demyelinization of the nervous system have also been observed.

Case presentation: A 37-year-old African woman with HIV infection diagnosed at 13 years of age was admitted to the emergency department after experiencing backache, severe headache, acute aphasia and psychomotor slowing for one week. Nine weeks earlier, highly active antiretroviral therapy in this patient had been changed because of loss of efficacy, and a rapid increase in CD4+ cell count and decrease of HIV viral load were observed. Magnetic resonance imaging of the brain showed extensive white matter lesions, and analysis of cerebrospinal fluid revealed an immunoreactive syndrome. Intensive investigations detected no opportunistic infections. A salvage therapy, including osmotherapy, corticosteroids and treatment of epileptic seizures, was performed, but the patient died from brainstem herniation 48 hours after admission. Neuropathologic examination of the brain revealed diffuse swelling, leptomeningeal infiltration by CD8 cells and enhancement of perivascular spaces by CD8+ cells.

Conclusion: Immune reconstitution inflammatory syndrome in this form seems to represent a severe autoimmunologic disease of the brain with specific histopathologic findings. This form of immune reconstitution inflammatory syndrome did not respond to therapy, and extremely rapid deterioration led to death within two days. Immune reconstitution inflammatory syndrome may also occur as severe leukoencephalopathy with fulminant cerebral edema during HIV infection with rapid immune reconstitution.
\end{abstract}

\section{Introduction}

The benefit of highly active antiretroviral therapy (HAART) in HIV-infected patients is the restoration of the immune system. According to the literature, up to $37 \%$ of these patients may develop an immune reconstitution inflammatory syndrome (IRIS) mainly when HAART is started in antiretroviral-naïve patients, who

* Correspondence: chrisoel@uni-muenster.de

1 University Hospital Muenster, Department of Neurology, A.-Schweitzer-Str. 33, D-48129 Muenster, Germany

Full list of author information is available at the end of the article develop a rapid recovery of immune function [1-4]. IRIS may develop based on opportunistic infections (e.g., with Mycobacterium tuberculosis, Cryptococcus neoformans or JC-virus inducing progressive multifocal leukoencephalopathy). IRIS of the peripheral or central nervous system as Guillain-Barré syndrome or leukoencephalopathy has also been observed [1-6].

\section{Case presentation}

A 37-year-old African woman with HIV infection diagnosed at age 13 years was admitted to the emergency 
department after experiencing backache, severe headache, acute aphasia and psychomotor slowing for one week. Nine weeks before admission to the hospital, HAART had been changed in this patient from $500 \mathrm{mg} / \mathrm{d}$ of zidovudine, $300 \mathrm{mg} / \mathrm{d}$ of lamivudine and $200 \mathrm{mg} / \mathrm{d}$ of emtricitabine to the fixed preparation of lopinavir/ritona$\operatorname{vir}(800 \mathrm{mg} / 200 \mathrm{mg} / \mathrm{d}$ ) and saquinavir (2000 mg/d). HAART was necessary for 10 years in this patient and was started at a level of $255 \mathrm{CD}^{+}$cells $/ \mathrm{mm}^{3}$. Before admission to the hospital, the patient's $\mathrm{CD} 4^{+}$cell count had rapidly increased from 20 to 610 cells $/ \mathrm{mm}^{3}$, and her HIV-1 viral load decreased from 63,200 to $120 \mathrm{c} / \mathrm{mL}$. Twelve hours after admission, the patient developed severe psycho-organic syndrome and repeated generalized epileptic seizures. Therefore, anticonvulsive therapy with levetiracetam was initialized. Computed tomography of the skull showed generalized cerebral edema without focal lesions. Intensive osmotherapy using mannitol and glycerol to minimize cerebral edema was performed. Blood tests revealed normal C-reactive protein and a normal white blood cell (WBC) count; radiographs of the chest were normal. Analysis of cerebrospinal fluid (CSF) showed pleocytosis $\left(36 \mathrm{WBC} / \mathrm{mm}^{3}\right)$, an elevated protein level of $1120 \mathrm{mg} / \mathrm{dL}$, a normal glucose level of $61 \mathrm{mg} / \mathrm{dL}$ and no oligoclonal bands. CSF microscopy yielded no bacteria or fungi. Virus polymerase chain reaction (PCR) results for JC-virus (JCV), herpes simplex virus (HSV), cytomegalovirus (CMV), Epstein-Barr virus (EBV), varicella zoster virus (VZV) and hepatitis B virus using whole blood were negative. PCR of the CSF did not detect JCV, HSV, CMV, EBV, VZV or Tuberculosis.

Twenty-four hours later, the patient fell comatose and needed mechanical ventilation. Magnetic resonance imaging (MRI) of the brain showed parietal and occipital hyperintense white matter lesions on T2-weighted images on both sides. Using contrast medium, these lesions did not reveal any enhancement in the T1weighted images. However, there was a strong enhancement in perivascular spaces (Figure 1). As salvage therapy, $1 \mathrm{~g} / \mathrm{d}$ of methylprednisolone and the antimicrobial agents ampicillin, acyclovir, ceftriaxone and fluconazole were applied. Without any improvement, the patient died from brainstem herniation two days after admission.

With the patient's relatives' consent, autopsy of the brain was performed. The post-mortem study of the brain showed a diffuse swelling of the whole brain and leptomeningeal infiltration with lymphocytes. These changes were also documented by MRI and represented the explanation for the epileptic seizures. Severe inflammatory infiltration in perivascular spaces by T-lymphocytes of the $\mathrm{CD}^{+}$subtype was found (Figure 2). Reactive astrocytes were found in the white matter, but no macrophages were seen. Results of Gram, periodic acid-Schiff and Grocott staining and immunocytochemistry for

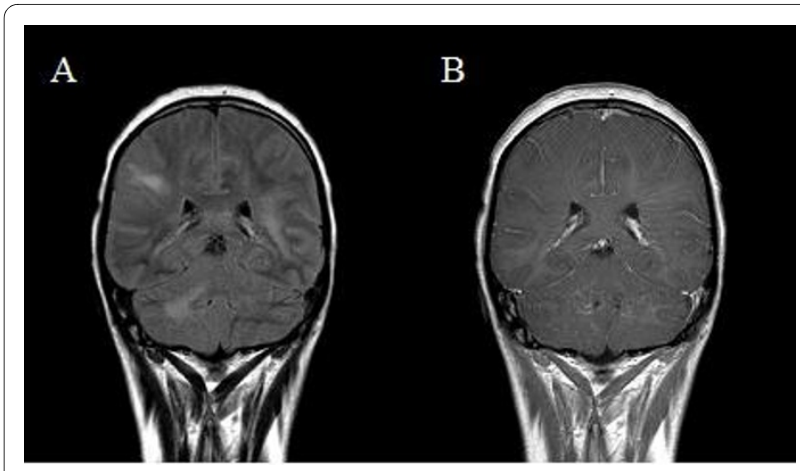

Figure 1 Magnetic resonance imaging (MRI) scans. This MRI scan from the patient's initial presentation shows a perivascular infiltration and intraparenchymal, large-scale lesions in both hemispheres on the T2-weighted images $(\mathbf{A})$. Application of gadolinium revealed no enhancement of the lesions in the T1-weighted images (B).

CMV and toxoplasma were negative. Congestion bleeding in the brainstem was the result of herniation, leading to death.

\section{Discussion}

A detailed case report of severe lethal leukoencephalopathy presumably an autoimmunologic manifestation of IRIS proven by typical neuropathologic results and corresponding MRI results is presented. IRIS usually starts after the initiation of HAART. The main risk factor for IRIS represents rapid immune restoration from a high viral load and low CD4+ cell count at the beginning of HAART [1-4,7]. Recovery of lost CD4 $4^{+}$cells initiates a complex immune cascade, inducing IRIS. CD4+ cells trigger cytotoxic reaction of $\mathrm{CD} 8^{+}$cells against antigens and may cause vasculitis, cerebritis or both $[4,8]$. The increase

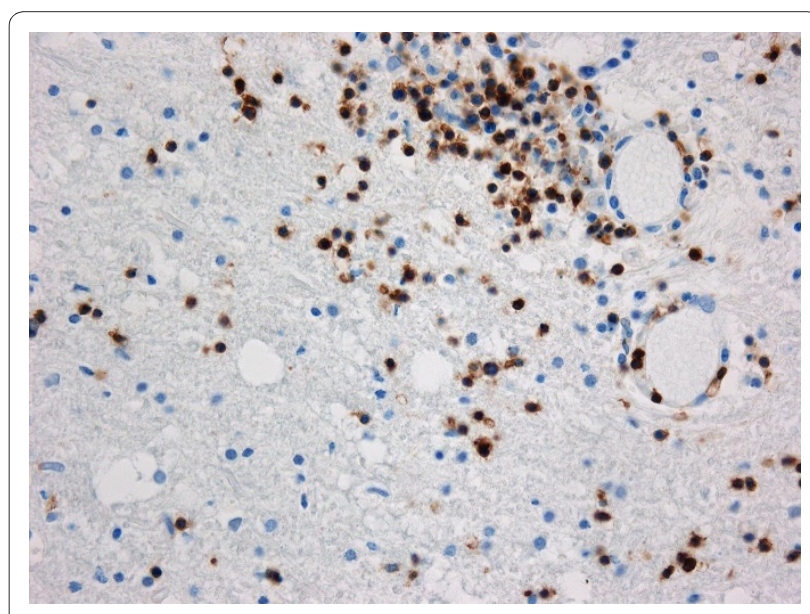

Figure 2 Histopathology. On histopathologic examination, lymphocytic infiltrates are encountered around cerebral blood vessels and in the white matter. Immunohistochemistry for CD3 confirms the T-cellular character of the inflammatory infiltrates (original magnification $\times 400$ ) 
in circulating newly developed $\mathrm{CD} 4{ }^{+}$cells triggers abnormal immune responses by $\mathrm{CD}^{+}$dysfunction in different organs such as the liver, eyes, lungs and brain $[1-4,8]$. IRIS is well known during opportunistic infections (e.g., toxoplasmosis, progressive multifocal leukoencephalopathy). IRIS manifesting as Guillain-Barré syndrome (GBS) or severe leucencephalopathy occurs only rarely [1,4-6,9]. Neuropathologic examination revealed perivascular infiltration of T-lymphocytes predominantly of the $\mathrm{CD}^{+}$ subtype in the brain and enhancement of perivascular spaces. Both pathologic findings have been described as irregular immune responses during IRIS [9]. Diffuse infiltrative lymphocytosis syndrome as an uncommon manifestation characterized by persistent circulating $\mathrm{CD}^{+}$ lymphocytosis in salivary glands may represent a less severe manifestation of this form of IRIS, and infiltration of a brain by $\mathrm{CD} 8^{+}$cells is proven in this case by neuropathologic examination $[5,8]$. Because no opportunistic infection could be detected, the autoimmunologic cause of IRIS is most likely and is demonstrated by neuropathologic results. Although wide pharmacologic therapy with corticosteroids and antimicrobial agents as salvage therapy was performed, the patient died two days after admission, showing the ineffectiveness of therapies that are generally effective in other forms of IRIS. The benefit of corticosteroid therapy has been described in patients with IRIS after opportunistic infection and is also an established therapy in patients with GBS $[1,3,10]$. In contrast to these results, corticosteroids were not successful in this fulminant form of IRIS presenting as severe, rapid, progressive leukoencephalopathy.

Up until now, diagnostic and therapeutic standards for IRIS have not been precise, and a clear classification and guidelines for therapeutic strategies in these new manifestations of IRIS are absolutely necessary. In every case, IRIS represents a severe complication of excellent immune restoration and should be considered in rapidly deteriorating patients. It represents a big diagnostic problem because predictors indicating early-stage IRIS are still unknown.

\section{Conclusion}

If distinct neurologic symptoms appear in HIV-1infected patients after initiation of HAART and initial clinical improvement, central nervous system IRIS should be considered. Application of corticosteroids represents a reasonable therapy for patients with mild cerebral edema but may fail in those with severe, rapid, progressive cases.

\section{Consent}

Written informed consent was obtained from the patient's next-of-kin for publication of this case report and any accompanying images. A copy of the written con- sent is available for review by the Editor-in-Chief of this journal.

\section{Competing interests}

The authors declare that they have no competing interests.

\section{Authors' contributions}

CO was responsible for the medical care of the patient and was the major contributor in writing the manuscript, RD was the head of the intensive care unit, $J \mathrm{M}$ was the responsible for the medical care in the intensive care unit, TN analyzed the radiologic examination, EBR is the department chair and IWH was the head of the division of neuro-AIDS and was a contributor in writing the manuscript.

All authors read and approved the final manuscript.

\section{Author Details}

1 University Hospital Muenster, Department of Neurology, A.-Schweitzer-Str. 33, D-48129 Muenster, Germany, 2University Hospital Muenster, Department of Internal Medicine, A.-Schweitzer-Str. 33, D-48129 Muenster, Germany and 3 University Hospital Muenster, Institute for Clinical Radiology, A.-Schweitzer-Str. 33, D-48129 Muenster, Germany

Received: 28 October 2009 Accepted: 17 July 2010

Published: 17 July 2010

\section{References}

1. Dhasmana DJ, Dheda K, Ravn P, Wilkinson RJ, Meintjes G: Immune reconstitution inflammatory syndrome in HIV-infected patients receiving antiretroviral therapy: pathogenesis, clinical manifestations and management. Drugs 2008, 68:191-208.

2. French MA: Disorders of immune reconstitution in patients with HIV infection responding to antiretroviral therapy. Curr HIV/AIDS Rep 2007, 4:16-21.

3. Shelbourne SA, Visnergawala F, Darcourt J, Graviss EA, Giordano TP, White AC Jr, Hamill RJ: Incidence and risk factors for immune reconstitution inflammatory syndrome during highly active antiretroviral therapy. AIDS 2005, 19:399-406.

4. Venkataramana A, Pardo CA, MCArthur JC, Kerr DA, Irani DN, Griffin JW, Burger $P$, Reich DS, Calabresi PA, Nath A: Immune reconstitution inflammatory syndrome in the CNS of HIV-infected patients. Neurology 2006, 67:383-388.

5. Knysz B, Bolanowski M, Klimczak M, Gladysz A, Zwolinska K: Graves' disease as an immune reconstitution syndrome in an HIV-1-positive patient commencing effective antiretroviral therapy: case report and literature review. Viral Immunol 2006, 19:102-107.

6. Teo EC, Azwra A, Jones RL, Gazzard BG, Nelson M: Guillain-BarréSyndrome following immune reconstitution after antiretroviral therapy for primary HIV infection. J HIV Ther 2007, 12:62-63.

7. Murdoch DM, Venter WDF, Feldmann C, van Rie A: Incidence and risk factors for the immune reconstitution inflammatory syndrome in HIV patients in South Africa: a prospective study. AIDS 2008, 22:601-610.

8. Feller C, White JP, Bouckaert M, Muthuphei MN: Diffuse infiltrative CD8+ lymphocytosis syndrome: review of the literature and report of a case. SADJ 2007, 62:314-317.

9. Vendrely A, Bienvenu B, Gasnault J, Thiebault JB, Salmon D, Gray F: Fulminant inflammatory leukencephalophathy associated with HAART-induced immune restoration in AIDS-related progressive multifocal leukencephalopathy. Acta Neuropathol 2005, 109:449-455.

10. Tan K, Roda R, Ostrow L, McArthur J, Nath A: PML-IRIS in patients with HIV infection. Clinical manifestations and treatment with steroids. Neurology 2009, 72:1458-1464.

doi: $10.1186 / 1752-1947-4-214$

Cite this article as: Oelschlaeger et al., Severe leukoencephalopathy with fulminant cerebral edema reflecting immune reconstitution inflammatory syndrome during HIV infection: a case report Journal of Medical Case Reports 2010, 4:214 